\title{
Predicting the misconception of dengue disease based on the awareness survey
}

\author{
Abrar Noor Akramin Kamarudin', Zurinahni Zainol², Nur Faeza Abu Kassim³ \\ ${ }^{1,2}$ School of Computer Science, Universiti Sains Malaysia, Malaysia \\ ${ }^{3}$ School of Biological Sciences, Universiti Sains Malaysia, Malaysia \\ ${ }^{3}$ Vector Control Research Unit, School of Biological Sciences, Universiti Sains Malaysia, Malaysia
}

\begin{tabular}{l} 
Article Info \\
\hline Article history: \\
Received Nov 20, 2019 \\
Revised Mar 29, 2020 \\
Accepted Apr 3, 2020 \\
\hline
\end{tabular}

\section{Keywords:}

Attitudes

Awareness survey

Dengue

Machine learning

Misconception prediction

Personalization

\begin{abstract}
Mosquito-borne disease such as dengue fever is a pervasive public health problem around the world and further investigation is needed to rectify the misunderstanding of the disease among communities. This requires a personalized information delivery, which will effectively fix the problem. The process of personalizing information requires several major steps: (i) determine the attributes which will be used to interpret a person, (ii) selects an algorithm which will accurately and efficiently classify the person according to the retrieved background information, and (iii) recommends the correct information to rectify the particular misunderstanding. This research paper considers the first two steps. First, data regarding the knowledge, attitudes and prevention practices are determined from the established literature where some variables give a significant impact on the predictive model. In the second step, five performed machine learning algorithms were tested for the classification task. The result indicates that the use of support vector machine and decision tree algorithms provide the best performance in classifying the person's understanding regarding the dengue fever.
\end{abstract}

This is an open access article under the CC BY-SA license.

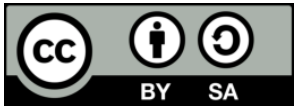

\section{Corresponding Author:}

Zurinahni Zainol, School of Computer Science, Universiti Sains Malaysia, 11800 Georgetown, Penang, Malaysia. Email: zuri@usm.my

\section{INTRODUCTION}

The rate of mosquito-borne diseases (MBDs) outbreak is always increased every time the seasons change due to various factors such as socioeconomic and environmental changes, socio-geographical, imported cases and human movement patterns [1,2]. All of the elements can continue the mosquito propagation and increase the difficulties of controlling the MBDs as predicting the future consequences in a changing environment demands a greater understanding of the array of biological and environmental features [3]. Current MBDs outbreak prediction systems were developed based on environmental factors such as flood, types of building, daily mean temperature, daily rainfall and vegetation index [4-7]. House Index and Breteau Index features were also often included in the predictive model to denote the risk areas [8]. Most studies have proven that climate change gives a significant impact on the increasing risk of dengue cases by using a data mining classification algorithm $[9,10]$. Obtaining such data requires constant observation around the hotspot area, but, the environmental monitoring system alone still cannot decrease the infection of dengue virus [1].

To improve the prediction framework, the morbidity rate forecasting works better when the vectors like Aedes aegypti and Aedes albopictus female mosquitoes and larvae index were selected as parameters as 
reported in [11]. Thus, this generates an idea to include the attributes based on the human behaviors regarding the MBDs as in [12-18] and put it into the predictive model to classify one's misconception in a personalized e-learning system. This research proposes the human knowledge, attitudes and preventive practices to be included in the machine learning approach in order to identify and provide a simple MBDs awareness program in a personalized e-learning environment. The proposed predictive framework for the personalized e-learning environment is illustrated in Figure 1.

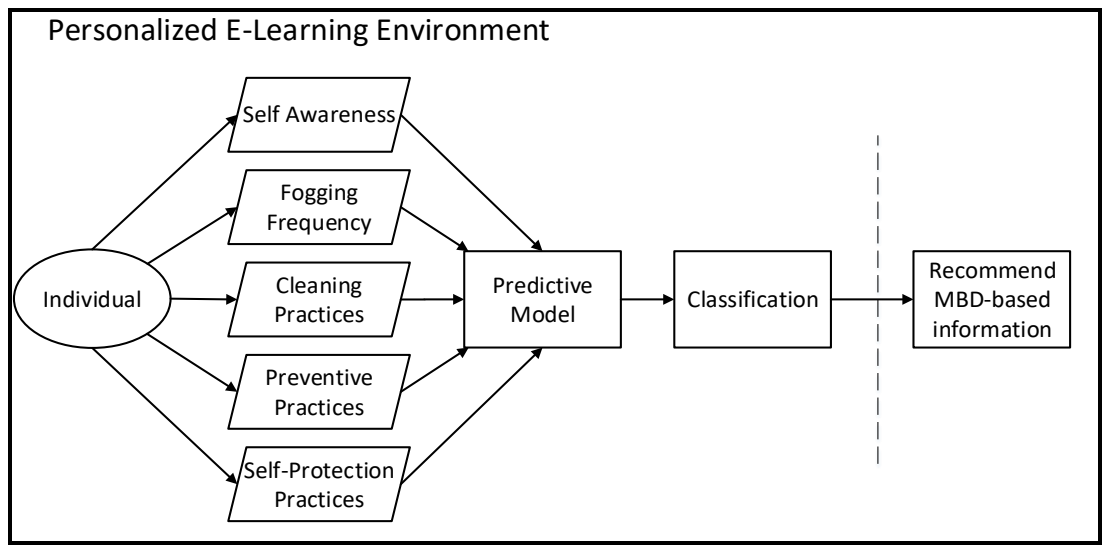

Figure 1. A predictive system to classify the user's misconception of MBDs in a personalized e-learning environment

To curb the MBDs particularly in hotspot areas, it is important to ensure that members of the public have adequate awareness through effective educational intervention. Social media usage such as Facebook and Twitter were the preferred source of dengue information among the public [19]. Health stakeholders of many countries often tweet and create a special posting to announce the epidemic outbreak of dengue in certain areas. This includes how to control the endemic and to provide early information about disease detection to the public. However, accessing such information might be hard for the people who lived in the rural and remote areas. Relevant MBDs-based information can be recommended to mold positive attitudes and educate the best preventive practices among the children. For instance, personal attributes and environmental factors such as region, specific location features and climate contexts were used in [20] to predict the human's risk of contracting infectious diseases. While in [21], an application is also made for the user to submit the information related to mosquito prevalence (e.g. felt, seen, bitten, heard mosquitoes). The public also can report the presence of Aedes that is a vector mosquito at the current location immediately and report dengue cases in the household or surrounding communities. This open application requires active citizen engagement and combined with other data, such as ovitrap egg counts and micro-climate data to propose the stakeholders proactively in disease prevention, control and education. However, the application might faces underreport when there is no participation from the people at certain places.

\section{RESEARCH METHOD}

Dengue knowledge, attitudes, and practices surveys have been conducted frequently to describe the communities' prevention towards the disease. It is expected that the knowledge of dengue and Aedes is high among the public due to the good risk practices for dengue and good risk perception that have been promoted through mainstream media and in education. An online community-based survey was carried out on the 1st September 2018 until 10th December 2018 with 640 respondents of the Malaysian public aged 7 years old and above. The questionnaire is posted and shared in a few Malaysian Facebook science-based pages and other groups.

The self-administered structured questionnaire covered all aspects of demographic profiles include gender, age, marital status, education level, and housing type. Concerning the awareness of the dengue issue, the participants need to answer general knowledge of mosquitoes such as the mosquito's breeding sites, mosquito's prevention method and mosquito's active biting time. Concerning to the awareness of dengue presence and prevention practices, the participants were asked to provide information regarding the fogging frequency in their living area. The fogging activity, in this context, was interpreted as their location might be a hotspot area or not. This follows a section on thirteen questions in the True/False setup relevant to cleanliness, trash management, water reservoir management and mosquito repellant usage. The logical flow 
of the questionnaire culminated as a part of the action by the study participants associated with the general knowledge of mosquito behavior.

The online survey is the fastest way to collect data as respondents' backgrounds are varied while keeping their anonymity. The items on the questionnaire were adapted from previously validated instruments with some modifications to meet the research objective. Close-ended questions are used to enhance the simplicity and easiness of analysis. Respondents just need to choose the best answer according to their existing knowledge.

Both descriptive analysis and machine learning were used in the experiment. Descriptive analysis was utilized to summarize the socio-demographic such as age, gender, marital status, ethnicity, highest education and type of residence. The machine learning algorithms were tested on 18 attributes of individual knowledge, attitudes and practices such as cleanliness; waste management; clogged drains and stagnant water handling; aerosol spray or mosquito repellent usage; cloth types and color usage, and the misconception of mosquito's breeding site, mosquito's active bite time and dengue disease transmission. Table 1 described the selected features that are found relevant to the proposed framework.

Table 1. Selected features mapping to the personalized e-learning environment

\begin{tabular}{|c|c|c|}
\hline No. & Features & Importance \\
\hline 2. & Fogging in housing area frequency & $\begin{array}{l}\text { To identify the availability of stagnant water that are } \\
\text { not disposed of properly or taken care of by the } \\
\text { communities as a potential breeding site. }\end{array}$ \\
\hline 5 . & Dumping trash that can hold water in the right place & different set of attitudes and practices of the cleaning \\
\hline 6. & Cleaning the drains or clogged waterways frequency & habit. \\
\hline 7. & Closed or discarded water reservoir before a long vacation & To identify the level of preventive practices where \\
\hline 8. & Checking larvae at the water reservoir & different demographics might have a different type \\
\hline 9. & Putting Abate/chemicals in water reservoirs to avoid larvae & of water management in their living space. \\
\hline 10. & Wearing long-sleeved shirts and long pants to prevent mosquito bites & To identify the level of self-protection practices as \\
\hline 14. & Sleeping in the mosquito net & \\
\hline 15. & Applying mosquito repellent cream on the body & \\
\hline
\end{tabular}

The machine learning algorithms training and testing were performed by using WEKA application, a data mining application developed at the University of Waikato in New Zealand. Different kinds of predictive models were built and the forecasting performances of those models were evaluated. The algorithms used in the model building are support vector machine (SVM), decision tree (DT), Logistic regression (LR), naïve bayes (NB) algorithm and artificial neural network (ANN). Tenfold cross-validation technique was utilized to obtain the accuracy of each algorithm in classification. Such classification algorithms comparison had been done to predict the chronic kidney disease (CKD) where naïve bayes performs the highest accuracy [22].

\section{RESULTS AND DISCUSSION}

There are 640 respondents who administered the online survey. When the gender ratio is concerned, the relative proportion of females respondents was higher $(80 \%)$ when compared with male participants Table $2.76 .4 \%$ of the respondents are aged 18 to 40 years old, $12.5 \%$ aged 7 to 17 years old and $11.1 \%$ aged more than 40 years old where more than $60 \%$ of them are married. The largest number of the respondents is coming from the Malay ethnic group (93.1\%) followed by others (Chinese, Indian, Dusun, Kadazan, Melanau, Bidayuh, Brunei, and Iban). Most of the respondents have attained tertiary education (73.5\%) followed by secondary school $(26 \%)$ and primary school $(0.5 \%)$. This illustrates that most respondents are well educated and should have minimal knowledge of MBDs especially dengue fever. It is also found that most of the respondents lived in a terrace or a twin house $(43.7 \%)$.

Amongst the survey participants, $91.9 \%$ of respondents declare that they are aware of the presence of Aedes mosquitoes in their area shown in Table 2. When asked about the frequency of fogging in their residential area, more than $10 \%$ of respondents reported that it always happens in their place. Over 50\% of respondents described that their house has medium to many plants, and near $70 \%$ of them are living out of the urban area. 
Table 2. Demographic information about participants surveyed

\begin{tabular}{lcc}
\hline \multicolumn{1}{c}{ Characteristics } & $\mathrm{N}$ & $\%$ \\
\hline Sex & & \\
$\quad$ Female & 512 & 80 \\
$\quad$ Male & 128 & 20 \\
Age (range) & & \\
$\quad 7-17$ & 80 & 12.5 \\
$18-40$ & 489 & 76.4 \\
$41+$ & 71 & 11.1 \\
Marital Status & & \\
$\quad$ Married & 409 & 63.9 \\
$\quad$ Single & 231 & 36.1 \\
Ethnicity & & \\
$\quad$ Malay & 591 & 93.1 \\
$\quad$ Others & 49 & 6.9 \\
Highest education & & \\
$\quad$ Primary school & 3 & 0.5 \\
$\quad$ Secondary school & 166 & 26 \\
Tertiary education & 470 & 73.5 \\
Types of residence & & \\
$\quad$ Flat/Apartment/Condo & 168 & 26.3 \\
Terrace/Twin House & 280 & 43.7 \\
Bungalow/Village house & 192 & 30 \\
\hline
\end{tabular}

The majorities of people surveyed have never experienced dengue fever; however, near $20 \%$ of them have gone through it before. It is found that $18 \%$ of respondents did not clean the area around their house and there might be a correlation to the rise of the dengue fever. Furthermore, it is also reported that the prevention practices among the respondents were very high, where $98.9 \%$ of them throw the trash in the right place.

It is found that more than $80 \%$ of respondents were disposing of the rubbish that can hold water in the right place; ensure the water reservoir is closed or discarded before a long vacation, and; always check the water reservoir to avoid larvae. Additionally, the number of other characteristics has varied where near $30 \%$ respondents have never cleaned the drains or clogged waterways; $28 \%$ of them did not use an aerosol spray to disperse mosquitoes at dark area; near $40 \%$ respondents did not use mosquito repellent such as liquids, coils, or trap in their living area; and only $25 \%$ respondents put abate or chemicals in water reservoirs to avoid mosquito larvae. Furthermore, near half of respondents wear long-sleeved shirts and long pants to prevent mosquito bites and wear bright-colored clothing to prevent mosquito bites. Only $3.9 \%$ of respondents used a mosquito net for sleeping and $11.4 \%$ apply the mosquito repellent cream to their body.

Although $91.1 \%$ of respondents declared that they were aware of the Aedes mosquito's appearance, there were $67 \%$ of respondents who cannot answer the three questions correctly as illustrated in Table 3. Thus, they are classified as having a misconception of the MBDs issue. The previous study shows the majority of respondents just finished secondary school and only $54.6 \%$ of them have a high level of knowledge regarding the dengue infections [15]. This result indicates that the current number of misconceptions is very high among the public although they are highly educated. Respondents might think they have sufficient knowledge regarding the dengue through the information they obtained from the television, newspaper, radio, social media, and awareness campaign, but that might not comprehend or half delivered where more than half of them are found unable to answer the questions correctly. Therefore, it is very important for the education stakeholders to include the prediction system into their e-learning system to identify the target users and increase their MBDs awareness in a personalized manner.

In order to correctly identify a person's understanding regarding the MBDs issue, the performance of selected machine learning algorithms was evaluated. The selected algorithms will be used for training and testing to classify the person whether they need further intervention based on the 18 features as listed in Tables 3 and 4. The true-positive (TP) rate, false-positive (FP) rate, precision, recall, F-measure, and time took to build the classifier are presented in Table 5 for each algorithm. It is found that the SVM and DT are the most precise algorithms to classify the person's misunderstanding with the precision of 0.994 and 0.008 false-positive rate. However, DT took lesser time than SVM to classify due to the input type was categorical and not continuous. Surprisingly, ANN performs inferior to the logistic regression model as the model-building process is less complicated for logistic regression, and may be considered not worthy of the artificial neural networks. In fact, the ANN model can be seen as nonlinear generalizations of logistic regression, and thus at least as powerful as that model which in this case, it was not significantly better to use neural networks.

Based on the result, the DT algorithm is found suitable to be used in the predictive model to support the objective of the research. Traditional dengue fever classification by using Naïve Bayes algorithm has 
been conducted in [23] to identify positive or negative results, based on several attributes such as fever, bleeding, flu, myalgia, and other symptoms with the precision of 0.92. As in [24], the DT algorithm has been used to predict patients with severe dengue that may warrant admission based on several variables (e.g. vomiting, pleural effusion, and systolic blood pressure) which return precision rate up to 0.81 . It is also has been used in [25] to propose a patient-monitoring plan and outpatient management of fever in resource-poor settings by using the clinical features and laboratory indicators to identify the severity of the dengue cases.

A study suggests the increase of knowledge through social media can influence the positive attitude and practices of dengue prevention measures [26]. However, social media alone cannot be personalized to fulfill the criteria as human attitude regarding the MBDs was not taken into consideration. This study is proposing the misconception of the disease can be identified among the public in the form of a quiz upon using the e-learning system. Once the system obtained enough information regarding the user's awareness background, it will analyze and personalized the relevant information regarding the MBDs between the learning sessions. The information is also can be fed directly to the target user, such as the children. His can be done based on a few rules where the children might be at a very young age of using the Internet or the children who lived in rural areas. Otherwise, feeding such information to a non-target user will be wasting the resources. Further investigation is needed to identify the differences of culture, socioeconomic background, and location which may also contribute to the preventive practices among the children. Thus, this paper highlights the big opportunity for the health authorities and education stakeholders to promote such campaigns through e-learning systems or any available open learning platform.

Table 3. Awareness of dengue presence and prevention practices

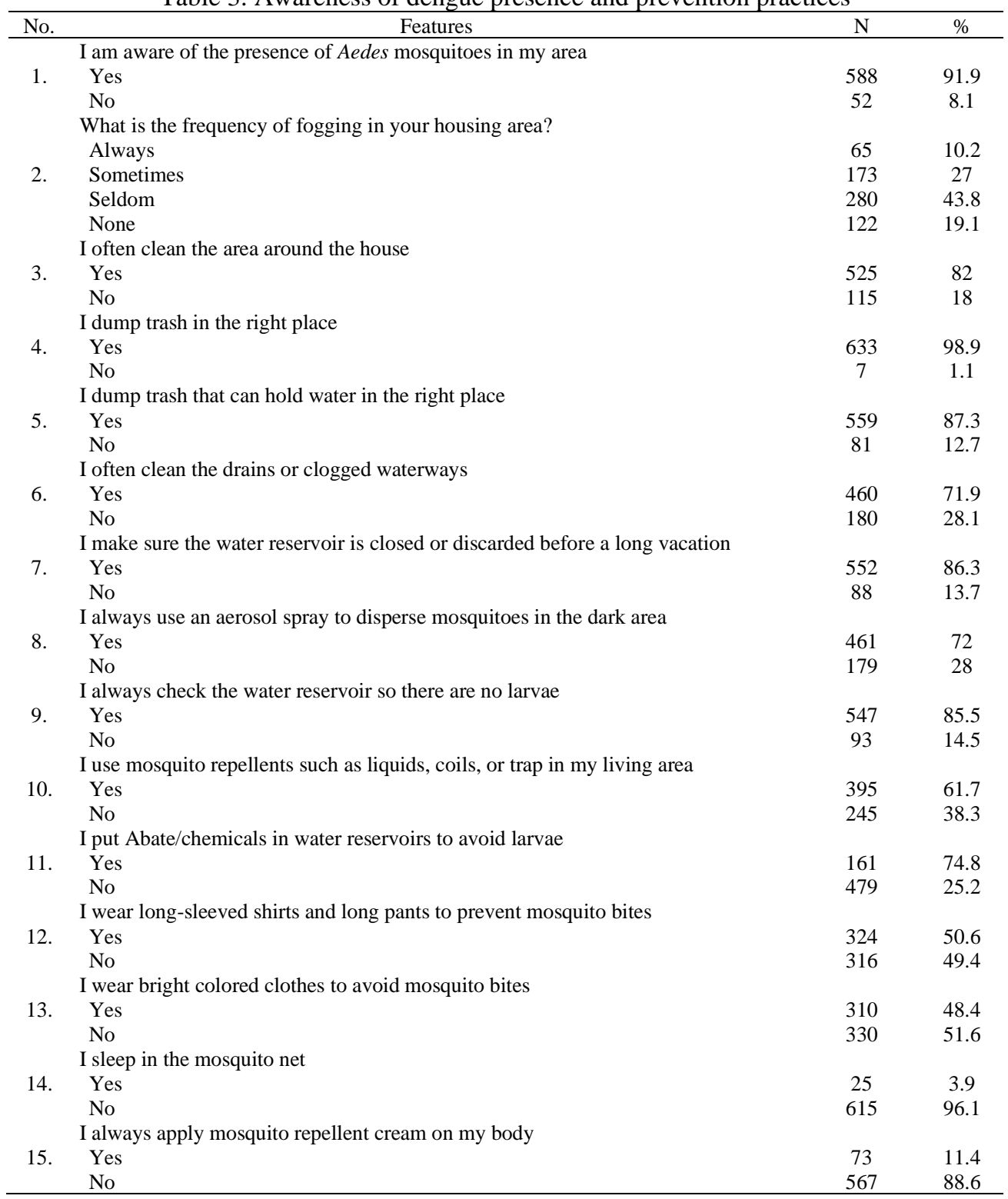

Predicting the misconception of dengue disease based on... (Abrar Noor Akramin Kamarudin) 
Table 4. Knowledge of Aedes characteristics

\begin{tabular}{|c|c|c|c|}
\hline No. & Questions & $\mathrm{N}$ & $\%$ \\
\hline 1. & $\begin{array}{l}\text { I know Aedes mosquito breeding in the following areas: } \\
\text { Stagnant water } \\
\text { Septic tank } \\
\text { Drain with waste } \\
\text { Container with clear water }\end{array}$ & & \\
\hline 2. & $\begin{array}{l}\text { I know Aedes mosquitoes actively bites at the following times: } \\
\text { Dawn } \\
\text { Afternoon } \\
\text { Evening } \\
\text { Night }\end{array}$ & & \\
\hline 3. & $\begin{array}{l}\text { How is dengue transmitted? } \\
\text { Mosquito bites } \\
\text { Contaminated and exposed foods } \\
\text { Don't know }\end{array}$ & & \\
\hline & Correctly answer all questions & 211 & 33 \\
\hline & Incorrectly answer some/all questions & 429 & 67 \\
\hline
\end{tabular}

Table 5. Performance comparison of the classification algorithms

\begin{tabular}{ccccccc}
\hline Algorithms & TP rate & FP rate & Precision & Recall & F-measure & Time taken (second) \\
\hline NB & 0.991 & 0.009 & 0.991 & 0.991 & 0.991 & 0.01 \\
LR & 0.991 & 0.012 & 0.991 & 0.991 & 0.991 & 0.24 \\
ANN & 0.986 & 0.012 & 0.986 & 0.986 & 0.986 & 6.32 \\
SVM & 0.994 & 0.008 & 0.994 & 0.994 & 0.994 & 0.16 \\
DT & 0.994 & 0.008 & 0.994 & 0.994 & 0.994 & 0.05 \\
\hline
\end{tabular}

\section{CONCLUSION}

Based on the awareness survey, the majority of respondents applied good practices in dengue preventive measures. An effective prediction model to detect the MBDs misconception is still needed to identify the communities' awareness and enhance their knowledge by recommending information about dengue prevention and early symptom recognition. A predictive system should be embedded to analyze the user's behavior and provide personalized information. Several features that have been selected for the study are found relevant for the proposed framework. This research proposes the Decision Tree algorithm in the predictive model construction of the online dengue awareness program by taking into account the human behavior and their current knowledge regarding the MBDs. The research focuses on Aedes and dengue transmission due to the high number of dengue fever and dengue hemorrhagic fever cases in Malaysia compared to other MBDs. Considerably more work is needed to identify features and causes of other MBDs such as Malaria, Zika, and Chikungunya including geospatial analysis and environmental factors.

\section{ACKNOWLEDGEMENTS}

The first author would like to acknowledge the USM Fellowship Scheme for providing a scholarship to conduct the research.

\section{REFERENCES}

[1] R. Jain, S. Sontisirikit, S. Iamsirithaworn, and H. Prendinger, "Prediction of dengue outbreaks based on disease surveillance, meteorological and socio-economic data," BMC infectious diseases, vol. 19, no. 1, pp. 1-16, 2019.

[2] P. E. Parham et al., "Climate, environmental and socio-economic change: Weighing up the balance in vector-borne disease transmission," Philosophical Trans. of the Royal Soc. B: Biological Sci., vol. 370, no. 1665, pp. 1-17, 2015.

[3] W. J. Tabachnick, "Challenges in predicting climate and environmental effects on vector-borne disease episystems in a changing world," Journal of Experimental Biology, vol. 213, no. 6, pp. 946-954, 2010.

[4] S. Deb, C. M. L. Acebedo, G. Dhanapal, and C. M. C. Heng, "An ensemble prediction approach to weekly Dengue cases forecasting based on climatic and terrain conditions," J. of Health and Soc. Sci., vol. 2, no. 3, pp. 257-272, 2017.

[5] R. Zaki et al., "Public perception and attitude towards dengue prevention activity and response to dengue early warning in Malaysia," PLoS One, vol. 14, no. 2, pp. 1-22, 2019.

[6] M. K. Othman and M. S. N. M. Danuri, "Proposed conceptual framework of dengue active surveillance system (DASS) in Malaysia," 2016 International Conference on Information and Communication Technology, pp. 90-96, 2017.

[7] B. Modu, N. Polovina, Y. Lan, S. Konur, A. Asyhari, and Y. Peng, "Towards a predictive analytics-based intelligent malaria outbreak warning system," Applied Sciences, vol. 7, no. 8, pp. 1-20, 2017.

[8] H. L. Lee et al., "Dengue vector control in Malaysia-challenges and recent advances," IIUM Medical Journal Malaysia, vol. 14, no. 1, pp. 11-16, 2015. 
[9] J. K. Davis, G. Vincent, M. B. Hildreth, L. Kightlinger, C. Carlson, and M. C. Wimberly, "Integrating environmental monitoring and mosquito surveillance to predict vector-borne disease: Prospective forecasts of a west nile virus outbreak," PLoS Currents, vol. 9, 2017.

[10] A. L. V. Sharma, A. Kumar, L. Panat, and G. Karajkhede, "Malaria outbreak prediction model using machine learning," International Journal of Advanced Research in Computer Engineering \& Technology, vol. 4, no. 12, pp. 4415-4419, 2015.

[11] K. Kesorn et al., "Morbidity rate prediction of dengue hemorrhagic fever (DHF) Using the support vector machine and the aedes aegypti infection rate in similar climates and geographical areas," PLoS One, vol. 10, no. 5, 2015.

[12] A. Itrat et al., "Knowledge, awareness and practices regarding dengue fever among the adult population of dengue hit cosmopolitan," PloS one, vol. 3, no. 7, pp. 1-6, 2008.

[13] M. M. T. Aung et al., "Knowledge, attitude, practices related to dengue fever among rural population in Terengganu, Malaysia," Malaysian Journal of Public Health Medicine, vol. 16, no. 2, pp. 15-23, 2016.

[14] S. Boonchutima, K. Kachentawa, M. Limpavithayakul, and A. Prachansri, "Longitudinal study of Thai people media exposure, knowledge, and behavior on dengue fever prevention and control," Journal of Infection and Public Health, vol. 10, no. 6, pp. 836-841, 2017.

[15] F. M. Yussof, A. Hassan, T. Zin, T. M. A. R. Hussin, N. Kadarman, and R. Umar, "Knowledge of dengue among students in universiti sultan zainal abidin (Unisza), Terengganu, Malaysia and the Influence of knowledge of dengue on attitude and practice," Journal of Fundamental and Applied Sciences, vol. 2017, no. 2S, pp. 199-216, 2017.

[16] S. Jeelani, S. Sabesan, and S. Subramanian, "Community knowledge, awareness and preventive practices regarding dengue fever in Puducherry-South India," Public Health, vol. 129, no. 6, pp. 790-796, 2015.

[17] M. M. Mathania, S. I. Kimera, and R. S. Silayo, "Knowledge and awareness of malaria and mosquito biting behaviour in selected sites within Morogoro and Dodoma regions Tanzania," Malaria journal, vol. 15, no. 1, pp. 1-9, 2016.

[18] M. Dhimal et al., "Knowledge, attitude and practice regarding dengue fever among the healthy population of highland and lowland communities in Central Nepal," PLoS One, vol. 9, no. 7, pp. 1-15, 2014.

[19] W. R. W. Rosli, S. A. Rahman, J. K. Parhar, and M. I. Suhaimi, "Positive impact of educational intervention on knowledge, attitude, and practice towards dengue among university students in Malaysia," Journal of Public Health, vol. 27, no. 4, pp. 461-471, 2019.

[20] R. A. Vinarti and L. M. Hederman, "A personalized infectious disease risk prediction system," Expert Systems with Applications, vol. 131, pp. 266-274, 2019.

[21] A. N. Babu et al., "Smartphone geospatial apps for dengue control, prevention, prediction, and education: MOSapp, DISapp, and the mosquito perception index (MPI)," Envi. Monitoring and Assessment, vol. 191, no. 2, p. 393, 2019.

[22] Z. Saringat, A. Mustapha, R. D. R. Saedudin, and A. A. Samsudin, "Comparative analysis of classification algorithms for chronic kidney disease diagnosis," Bulletin of Elec. Eng. and Inf., vol. 8, no. 4, pp. 1496-1501, 2019.

[23] K. Shaukat Dar and S. M. Ulya Azmeen, "Dengue fever prediction: A data mining problem," Journal of Data Mining in Genomics \& Proteomics, vol. 06, no. 3, pp. 1-5, 2015.

[24] J. Tamibmaniam, N. Hussin, W. K. Cheah, K. S. Ng, and P. Muninathan, "Proposal of a clinical decision tree algorithm using factors associated with severe dengue infection," PLoS One, vol. 11, no. 8, pp. 1-10, 2016.

[25] K. Phakhounthong et al., "Predicting the severity of dengue fever in children on admission based on clinical features and laboratory indicators: Application of classification tree analysis," BMC pediatrics, vol. 18, no. 1, pp. 1-9, 2018.

[26] W. R. Wan Rosli, S. Abdul Rahman, J. K. Parhar, and M. I. Suhaimi, "Positive impact of educational intervention on knowledge, attitude, and practice towards dengue among university students in Malaysia,” Journal of Public Health, 2018.

\section{BIOGRAPHIES OF AUTHORS}

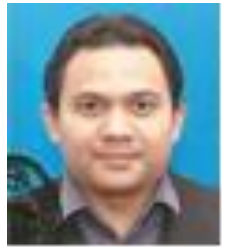

Abrar Noor Akramin Kamarudin is a Ph.D. student at the School of Computer Science, Universiti Sains Malaysia. His research is focused on vector-borne disease prediction as well as personalizing the education for the community.

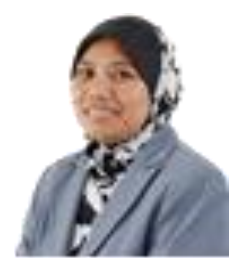

Zurinahni Zainol is an Associate Professor at the School of Computer Science, Universiti Sains Malaysia. Her research specialization is Theory of Database Design and Management, XML Document Schema Design, Data Model, and Software Development using Formal Specification.

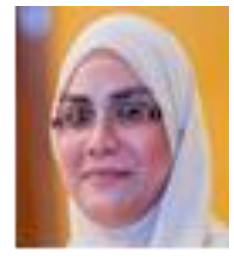

Nur Faeza Abu Kassim is an Associate Professor at the School of Biological Science, Universiti Sains Malaysia. She is an expert in medical and epidemiological entomology. Her research specialization is mosquito-borne disease $\&$ the role of vectors in transmission disease. Development of the integrated vector management, mosquito control monitoring/surveillance program, attractant and trapping system. 